\title{
Nature, Strength, and Cooperativity of the Hydrogen-Bonding Network in $\alpha$-Chitin
}

\author{
Volker L. Deringer, ${ }^{\dagger}$ Ulli Englert, ${ }^{* \dagger}$ and Richard Dronskowski* ${ }^{*}, \dagger$ \\ ${ }^{\dagger}$ Institute of Inorganic Chemistry and ${ }^{\ddagger}$ Jülich-Aachen Research Alliance (JARA-HPC), RWTH Aachen University, Landoltweg 1, \\ 52056 Aachen, Germany
}

\section{Supporting Information}

ABSTRACT: Chitin is an abundant biopolymer that stabilizes the exoskeleton of insects and gives structure to plants. Its macroscopic properties go back to an intricate network of hydrogen bonds that connect the polymer strands, and these intermolecular links have been under ongoing study. Here, we use atomistic simulations to explore hydrogen bonding in the most abundant form, $\alpha$-chitin. The crystal structure exhibits disorder, and so discrete models are systematically derived as suitable approximants to the macroscopic material. These models then allow us to perform dispersion-corrected

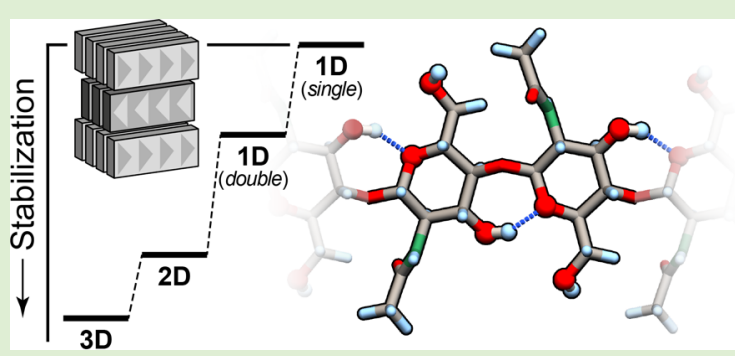
density-functional theory (DFT-D) simulations on the threedimensional crystal network and on lower-dimensional fragments. Thereby, we rationalize the nature of hydrogen bonding and the role of crystallographic disorder for the stability of $\alpha$-chitin, and complement previous, larger-scale molecular-dynamics (MD) simulations as well as recent fiber-diffraction experiments. Our results provide new, atomic-level insight into one of Nature's most abundant building materials, and the techniques and concepts are likely transferable to other biopolymers.

\section{INTRODUCTION}

Chitin, or poly- $N$-acetylglucosamine, is a fundamental biological macromolecule and an intriguing chemical compound. It ranks among the most abundant natural materials, being found in lobsters' claws, beetles' carapaces, fungi, and plants alike. ${ }^{1}$ The functionality and structural stability of chitin originates on the atomic scale; in particular, from strong hydrogen bonds (HBs) that connect the polymer strands. The atomic-scale arrangement of HBs in $\alpha$-chitin has been proposed in an initial structural study ${ }^{2}$ and recently refined based on cutting-edge synchrotron fiber X-ray diffraction (XRD). ${ }^{3}$ Hydrogen bonding in $\alpha$-chitin has also been probed by nuclear magnetic resonance $(\mathrm{NMR})^{4}$ and other experimental techniques (see ref 5 and references therein). Those secondary, noncovalent interactions are key to understanding (bio)organic crystal structures and furthermore they determine macroscopic properties such as stiffness and hardness. ${ }^{6}$ In turn, the attractive properties of biomaterials have inspired synthetic chemistry and have given rise to the burgeoning field of biomimetic materials science in which polysaccharides play a prominent role. ${ }^{7}$

How strong is hydrogen bonding in biomaterials? In principle, theoretical and computational chemistry can provide numerical answers to this question; see ref 8 for but one example. However, in many periodic systems (including chitin and other biopolymers), HBs form interwoven networks whose exploration is a nontrivial task. Furthermore, modeling extended crystal structures in most cases precludes the use of numerically expensive high-level wave function-based methods, at least for the time being. A range of approaches have been developed that do allow one to study $\mathrm{HB}$ networks in small- molecule crystal structures: energy-partitioning schemes such as PIXEL, ${ }^{9}$ analyses of the self-consistent electron density, ${ }^{10}$ or total-energy computations for crystals and lower-dimensional fragments. ${ }^{11}$ The latter technique is becoming increasingly accessible as economic, yet accurate computational methods are being developed and applied to molecular solids; for a recent perspective see, for example, ref 12 and references therein. Dispersion corrections to density functional theory (DFT-D) are prominent examples that we will discuss and use in this work.

Indeed, DFT-D computations have recently been applied to biomacromolecules as well, and they have prominently been used to study the related and even more abundant biopolymer, cellulose. $^{13}$ For example, the role of (directional) hydrogen bonding and (nondirectional) interchain dispersion were compared for different cellulose polymorphs. ${ }^{13 \mathrm{~b}}$ The usefulness of joint experimental-theoretical work was exemplified for two competing $\mathrm{HB}$ motifs in cellulose $\mathrm{I} \beta$, combining fiber neutron diffraction with Hartree-Fock and DFT energy minimization, and with molecular-dynamics (MD) simulations. ${ }^{14}$ Earlier already, simulations had been performed to dissect the $\mathrm{I} \beta$ crystal structure into lower-dimensional fragments, ${ }^{15}$ and the enzymatic deconstruction (or recalcitrance) of cellulose was studied by force-field $\mathrm{MD} .^{16}$ Theoretical investigations of isolated cellulose sheets were also reported recently. ${ }^{17}$

Received: December 8, 2015

Revised: January 29, 2016

Published: February 1, 2016 
The computational literature on chitin is somewhat more scarce but contains key contributions, too. Multiscale simulations of lobster cuticles (which are composed of $\alpha$ chitin) were performed by Nikolov et al., thereby spanning the length scales from quantum-mechanical (atomically resolved) computations to macroscopic properties. ${ }^{6 \mathrm{c}}$ As the $\mathrm{HB}$ arrangement in $\alpha$-chitin has been under scrutiny, a careful multiscale study of conformations and connectivity was reported by Petrov et al.; ${ }^{18}$ we will return to their work below. In another key study relevant to the present work, Beckham and Crowley applied force-field MD simulations to explore the distribution of HBs within $\alpha$-chitin nanocrystals, as well as enzymatic decrystallization pathways; ${ }^{19}$ this has been one of the first studies to investigate the role of $\mathrm{HB}$ patterns for the structural stability of $\alpha$-chitin. ${ }^{19}$ Finally, MD simulations have been concerned with interfaces between $\alpha$-chitin and protein models, which are again influenced by HBs to a significant degree. ${ }^{20}$

Unfortunately, other than for many (bio)molecular crystals, it is not trivial to directly translate the refined structure of $\alpha$ chitin into discrete quantum-chemical input. This is due to the presence of crystallographic disorder that gives rise to diverse HB patterns and connectivities, ${ }^{3,4}$ more diverse than can be modeled within a simulation cell of "only" a few hundred atoms. In a broader context, disorder and its effects are currently under very active study throughout chemistry and physics: ${ }^{21}$ understanding and controlling crystallographic disorder is of very practical importance in fields such as electronic memories, ${ }^{22}$ thermoelectrics, ${ }^{23}$ or hybrid perovskite solar cells. ${ }^{24}$ An even simpler representative of disordered materials, namely, hexagonal ice, has been atomistically studied using a combinatorial approach that seeks small, but manageable crystallographic cells in lieu of the "real-world" material. ${ }^{25}$ We will follow a comparable path in the present study.

In this work, we use periodic DFT-D to explore the nature of hydrogen bonding in $\alpha$-chitin. Our approach is somewhat perpendicular to previous theory studies: we do not aim to simulate entire fibrils or nanocrystals but restrict ourselves to the much smaller crystallographic unit cell, in turn gaining access to $a b$ initio (rather than force-field) accuracy. After having established structural models that take care of the aforementioned disorder, we compute interaction energies in $\alpha$ chitin, using dimensionally resolved analyses ${ }^{11 c, 26}$ whose scope is here seamlessly extended to biopolymers. Finally, one may ask about the cooperativity (or synergy) of HBs in solids: do they amplify each other, or do they merely coexist? ${ }^{27}$ Indeed, this question has been explicitly addressed for the cellulose allomorphs, ${ }^{28}$ and we will explore it for $\alpha$-chitin below. In a broader perspective, atomistic approaches as used here could provide useful complements to the many careful fiber diffraction studies of biomacromolecules, and they offer new and complementary insight into the physical and chemical nature of $\alpha$-chitin and related biomaterials.

\section{COMPUTATIONAL METHODS}

DFT computations were performed using the Perdew-BurkeErnzerhof (PBE) functional, ${ }^{29}$ corrected for dispersion interactions by the D3 scheme of Grimme and co-workers ${ }^{30}$ with Becke-Johnson (BJ) damping. ${ }^{31}$ The DFT-D3(BJ) approach was benchmarked before and was found suitable for hydrogen bonding and noncovalent interactions $\mathrm{s}^{32}$ as they are relevant here. For example, this particular method was tested against highly accurate, estimated CCSD $(\mathrm{T}) / \mathrm{CBS}$ energies for the $S 66 \times 8$ reference set, and adding the dispersion correction reduced the reported mean absolute error from 6.32 (PBE) down to $\left.1.46 \mathrm{~kJ} \mathrm{~mol}^{-1}(\mathrm{PBE}+\mathrm{D} 3(\mathrm{BJ}))\right)^{32 \mathrm{~b}}$ thereby reaching what may be dubbed "chemical accuracy" for molecular complexes. The situation for periodic solids is more delicate, but here as well, careful benchmarking revealed errors on the order of $4-5 \mathrm{~kJ} \mathrm{~mol}^{-1}$. ${ }^{32 \mathrm{c}}$ The suitability of DFT-D to treat polysaccharides has been noted earlier for the case of cellulose. ${ }^{13 a, b}$ We have furthermore validated this particular technique at the hand of the disorder-free allomorph $\beta$-chitin. For the latter, recent synchrotron $\mathrm{X}$-ray ${ }^{33 a}$ as well as neutron-diffraction data are available, ${ }^{33 \mathrm{~b}}$ and our computations reproduce the experimental lattice parameters very well to within $\sim 0.5 \%$ (Supporting Information). The more challenging quest, nonetheless, is to model the more abundant but crystallographically disordered $\alpha$-chitin, on which we focus in the present work.

All computations were performed with the Vienna ab initio Simulation Package (VASP 5.3.5), ${ }^{34}$ using hard (“'h") projector augmented-wave ${ }^{35}$ potentials and a plane-wave cutoff energy of 800 $\mathrm{eV}$. Initial structural relaxations minimized forces on atoms below $10^{-2}$ $\mathrm{eV} \AA^{-1}$; subsequently, final relaxations were done using very tight convergence criteria of $\Delta E<10^{-8}\left(10^{-6}\right) \mathrm{eV}$ cell $^{-1}$ for electronic (structural) cycles, respectively. Reciprocal space was sampled on $\Gamma$ centered Monkhorst-Pack grids, ${ }^{36}$ sized $4 \times 1 \times 2$ for the description of the conventional unit cell and reduced accordingly for supercell computations (see below).

\section{RESULTS AND DISCUSSION}

A Structural Model, or Three. The crystal structure of $\alpha$ chitin was recently revised based on fiber XRD (Figure 1) as

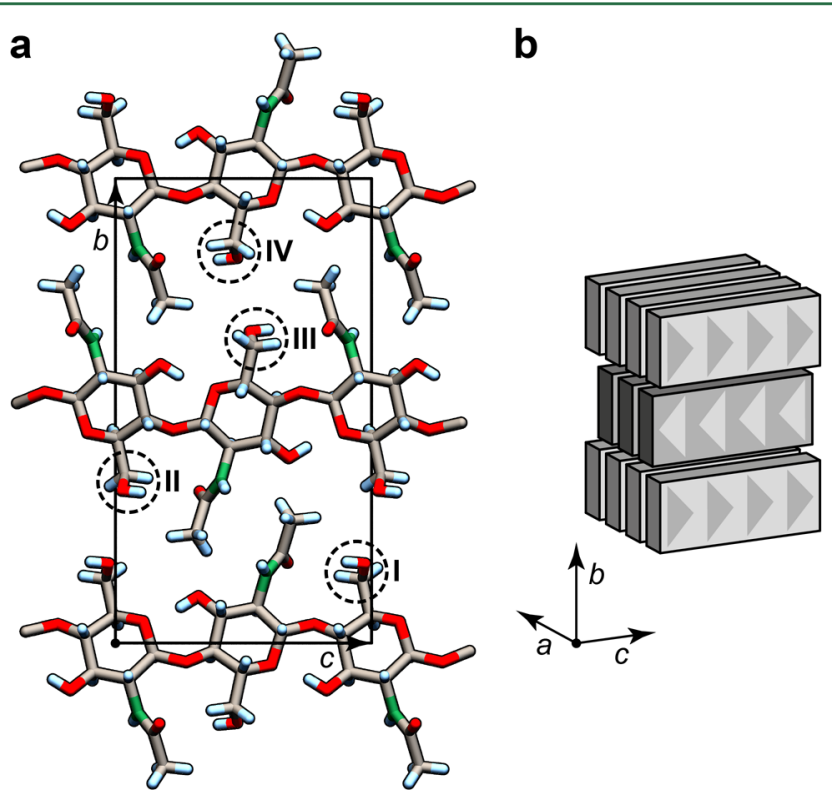

Figure 1. (a) Crystal structure of $\alpha$-chitin ${ }^{3}$ with polymer chains running along the $c$-axis. The hydroxymethyl groups in the unit cell have been highlighted and given Roman numerals. (b) Schematic drawing of how the chains are arranged in the crystal; arrows emphasize the antiparallel running direction.

reported in this journal; ${ }^{3}$ the authors' excellent structural data at $100 \mathrm{~K}$ serve as starting point for our study. There is, however, the aforementioned challenge of disorder; in $\alpha$-chitin, the hydroxymethyl $\left(-\mathrm{CH}_{2}-\mathrm{OH}\right)$ group can adopt more than one conformation, 3,18 and we label the two alternatives " $A$ " and "B" following Sikorski et al. ${ }^{3}$ (Figure 2). Refinement of the XRD data had arrived at occupation factors of $g_{\mathrm{A}}=0.58(8)$ and $g_{\mathrm{B}}=$ $0.42(8)$, respectively, ${ }^{3}$ which indicates a roughly equal occupation within experimental uncertainty. Hence, both hydroxymethyl configurations, $\mathbf{A}$ and $\mathbf{B}$, are expected to 

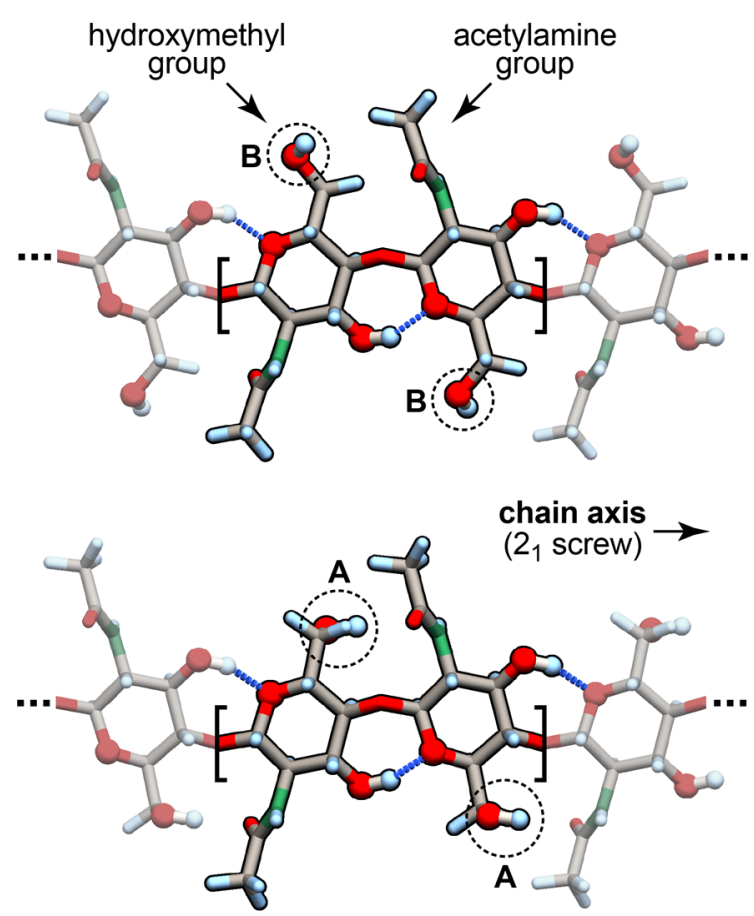

Figure 2. Chain motifs from optimized $\alpha$-chitin structure models, visualizing the repeat unit (in brackets; cf. Chart 1), the shortest $\mathrm{HB}$ contact within the chain (blue), as well as two possible orientations of the hydroxymethyl group (B/A). Note that the latter in A orientation may appear to also form additional HBs within the chain, but this is not the case; the hydroxymethyl and acetylamine moieties are tilted out of the paper plane in opposite directions. In the 3D structure, the hydroxymethyl group forms interchain $\mathrm{O}-\mathrm{H} \cdots \mathrm{O}$ bonds instead (see Figure $4 \mathrm{c}$ below).

contribute significantly to the macroscopic crystal, and neither of them can be neglected a priori. A comparable splitting between two configurations had also been identified in ${ }^{13} \mathrm{C}$ NMR experiments that suggested a 60:40 ratio. ${ }^{4}$ Finally, a more comprehensive and unconstrained conformational scan suggested that six out of seven hydroxymethyl groups do take one of the conformations proposed by Sikorski et al. (50\% A, 36\% B, $14 \%$ others $).{ }^{18}$ The restriction to "only" two conformations thus seems well justified both by experimental data and by theoretical results.

One unit cell of $\alpha$-chitin contains two chitobiose units (Chart 1) and thus four hydroxymethyl groups (Figure 1a) that we label as I-IV. Quantum-chemical input requires discrete coordinates for each of them-at variance with larger-scale MD-based simulations such as in ref 19 where statistical quantities are accessible and thus a percent "likelihood" of certain HBs forming can be derived (we will return to this below). In the present case, enumerating all possible discrete

Chart 1. Molecular Structure of Chitin (One Chitobiose Repeat Unit Is Shown)

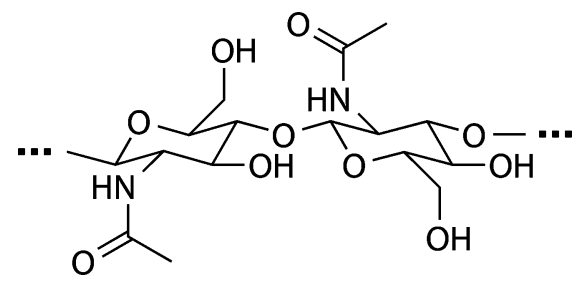

combinations leads to seven structural models distinguishable by symmetry; they are collected in Table 1 . The simplest ones

Table 1. Discrete Structural Models for $\alpha$-Chitin as Discussed in the Text and their Relative Stability $E_{\text {rel }}$ as Computed Using DFT-D ${ }^{a}$

$\begin{array}{ccc}\begin{array}{c}\text { O6 configuration } \\ \text { (sites I, II, III, IV) }\end{array} & \text { space group of model } & E_{\text {rel }}\left(\mathrm{kJ} \mathrm{mol}^{-1}\right) \\ \text { BBBB } & P 2_{1} 2_{1} 2_{1} & +18 \\ \text { ABBB } & P 1 & +12 \\ \text { AABB } & P 2_{1} & +12 \\ \text { ABAB } & P 2_{1} & +1 \\ \text { BAAB } & P 2_{1} & +3 \\ \text { AAAB } & P 1 & +2 \\ \text { AAAA } & P 2_{1} 2_{1} 2_{1} & \pm 0 \text { (reference) }\end{array}$

${ }^{a}$ Derived from the experimental data set of Sikorski et al. ${ }^{3}$ ("100 K.cif"). ${ }^{b}$ See Figures 1 a and 2 for illustration.

are obtained when all four hydroxymethyl groups have the same configuration, which is not expected in the macroscopic crystal but is important to study the limiting cases; let us denote these models as AAAA and BBBB. Both retain the initial spacegroup setting $\left(P 2_{1} 2_{1} 2_{1}\right)$. Mixed configurations such as $\mathbf{A B A B}$ or AAAB, on the other hand, require symmetry reductions to the subgroup $P 2_{1}$ or even to $P 1$. Those permutations that are not listed in Table 1 are equivalent to one of the existing entries (for example, BAAA is indistinguishable from AAAB). We have thus covered all possible discrete configurations within the constraints of the experimentally determined unit cell. These models are now amenable to quantum-chemical computations.

In a first step, the atomic coordinates in all candidate structures were fully optimized, while the cell vectors were locked to experimental values; ${ }^{3}$ these models were then compared energetically at the DFT-D level. Throughout this paper, we normalize all energies to one chitobiose unit (Chart 1). Setting the most stable configuration AAAA as the energy zero, three other models lie within a few $\mathrm{kJ} \mathrm{mol}^{-1}$ and thus are practically on par with the most stable one; the three remaining arrangements are less favorable. In no case, however, does the energy difference exceed $20 \mathrm{~kJ} \mathrm{~mol}^{-1}$; this is expected, as the mixed occupations both contribute significantly to the experimentally observed structure. This is different from cellulose $\mathrm{I} \beta$ in which one mode of hydrogen bonding is clearly favored over the other. ${ }^{14}$ In the following, we will focus on the following three models: on the two limiting cases, and furthermore on the intermediate structure $\mathbf{A B A B}$, which is the most favorable among the "mixed" models and allows us to study alternating hydroxymethyl group conformations within the polymer chains.

Next, it is important to test how the lattice parameters of $\alpha$ chitin result from a full DFT-D optimization; the computational technique is able to predict lattice parameters for noncovalently bonded solids with high accuracy, ${ }^{32 c}$ and thus if significant deviations would be observed this could indicate an intrinsic shortcoming of the constructed models. For the three $\alpha$-chitin models derived above, energy-volume curves were thus computed, used to fit the Birch-Murnaghan equation of state $^{37}$ and a final relaxation was done at the so-determined optimum volume. Table 2 compares the results to previous experimental data. The $c$-axis, along which the chains and thus the strong covalent bonds extend, is reproduced well, and the computed $c$ parameters for the various models differ by $<0.3 \%$ 
Table 2. Unit-Cell Parameters and DFT-D Computed Relative Energies of Fully Relaxed $\alpha$-Chitin Models

$\begin{array}{llllll} & & a(\AA) & b(\AA) & c(\AA) & E_{\text {rel }}\left(\mathrm{kJ} \mathrm{mol} \mathbf{m}^{-1}\right) \\ \text { expt } & \text { XRD }(\text { r. t. })^{2} & 4.74(1) & 18.86(2) & 10.32(2) & \\ & \text { XRD }(100 \mathrm{~K})^{3} & 4.715(80) & 18.78(17) & 10.33(10) & \\ \text { DFT-D } & \text { BBBB } & 4.722 & 18.40 & 10.36 & +17 \\ & \text { ABAB }^{a} & 4.730 & 18.69 & 10.37 & \pm 0 \\ & \text { AAAA } & 4.677 & 18.74 & 10.34 & \pm 0(\text { reference })\end{array}$

${ }^{a}$ The cell angles changed to $\alpha=90.0^{\circ}, \beta=92.6^{\circ}, \gamma=90.0^{\circ}$ due to the discrete structural model and the lowering of symmetry. In all other cases, $\alpha=$ $\beta=\gamma=90^{\circ}$.

among each other. The small size of this difference is easily understood, as the hydroxymethyl groups are located in the polymer's periphery and point away from the running direction of the chain. For the two perpendicular directions, in which the "interchain" forces dominate, the computed lattice parameters accordingly scatter more between the discrete models, by $1.1 \%$ ( $a$-axis) and $1.8 \%$ ( $b$-axis) at most. All three entries in Table 2, nonetheless, agree appreciably with experiments, especially given a certain error bar intrinsic to fiber diffraction. ${ }^{3}$ In other words, there is no significant departure from experimental observations, which once more points toward the suitability of the modeling approach used here.

Energetically, the fully optimized $\mathbf{A B A B}$ model is degenerate with AAAA (Table 2) within any reasonable accuracy limits, while BBBB is less favorable once more. Given that an a priori choice of "best" model is not possible, and their side-by-side comparison will be key in the discussion below, we will from now use structures optimized at the experimental lattice parameters, as done before in methodologically comparable studies. ${ }^{11 c, 26 a}$ That this procedure is well justified is best confirmed by the computed total energies of fully relaxed ABAB and AAAA; both differ by less than $1 \mathrm{~kJ} \mathrm{~mol}^{-1}$ from the respective result obtained using the experimental lattice vectors.

Geometric Criteria. With suitable models now constructed, we are able to derive structural parameters of HBs in $\alpha$-chitin. We first focus on the two shortest contacts, neither of which directly involves the hydroxymethyl group: the "intrachain" $\mathrm{O}-$ $\mathrm{H} \cdots \mathrm{O}$ bond (marked in blue in Figure 3), and the "interchain" $\mathrm{N}-\mathrm{H} \cdots \mathrm{O}$ contact that links adjacent acetylamine groups (green in Figure 3). Computed distances for both are given in Table 3; they refer to DFT-D relaxations with experimental cell vectors, but the interatomic distances are similar within $<0.01 \AA$ using fully optimized cells. We will later in this work add mediumrange $\mathrm{HBs}$ to the picture, but we will deliberately not look at HBs with very large $\mathrm{H}$-..acceptor distances, as it has been argued that at larger interatomic distances more nondirectional interactions will prevail. ${ }^{26 a, 38}$

We start by noting a visible difference between the two previous measurements in Table 3; both HBs seem longer in the recently refined structural model. This need not be a shortcoming of either experiment but may stem from a range of factors, including the collection of $\alpha$-chitin samples from different biological species that Petrov at al. pointed out convincingly. ${ }^{18}$ In this light, the conventionally defined donor $\cdots$ acceptor distances ( $\mathrm{O} \cdots \mathrm{O}$ and $\mathrm{N} \cdots \mathrm{O}$, respectively) are well reproduced by theory, and almost all of them reside within the range of experimental values. On purpose, we have not included experimental $\mathrm{H} \cdots \mathrm{O}$ distances because hydrogen atom localization in fiber diffraction is troublesome, as carefully discussed by Sikorski et al. themselves. ${ }^{3}$ We have tried to normalize the experimental hydrogen positions using default $\mathrm{O}-\mathrm{H}$ and $\mathrm{N}-\mathrm{H}$ bond lengths but this did not lead to

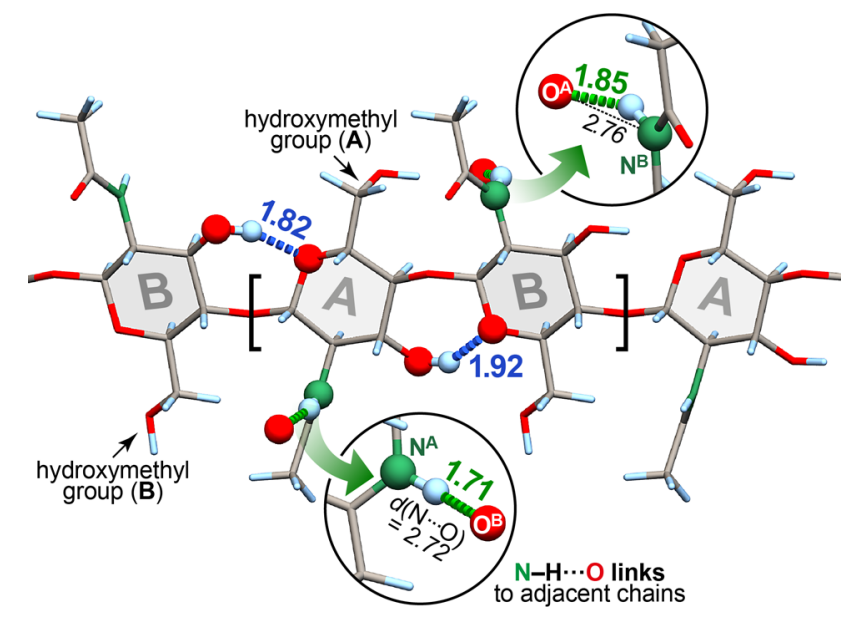

Figure 3. Fragment from the mixed "ABAB" model after DFT-D relaxation. The hydroxymethyl groups within a chain take alternating configurations, and the glycosidic rings have been labeled accordingly. The two shortest HBs in $\alpha$-chitin (Table 3) are emphasized in this viewgraph: the intrachain $\mathrm{O}-\mathrm{H} \cdots \mathrm{O}$ contact (blue) and the interchain $\mathrm{N}-\mathrm{H} \cdots \mathrm{O}$ bonds (green) that link the chain to adjacent ones (magnified in the insets; one neighboring acceptor oxygen is shown in both cases). All distances are given in $\AA$.

Table 3. Structural Parameters for the Shortest Hydrogen Bonds in $\alpha$-Chitin, from Experiments and Theory

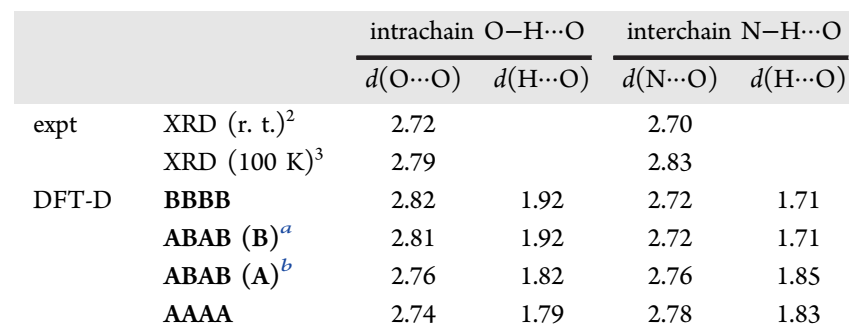

${ }^{a} \mathrm{HB}$ acceptor near a "B" hydroxymethyl group, thus locally resembling the BBBB structure; see Figure $3 .{ }^{b} \mathrm{HB}$ acceptor near an "A" hydroxymethyl group.

satisfactory HB angles; indeed, a "custom-fitting” optimization is required here instead. ${ }^{39}$

Looking more closely at the modeling results, an indirect influence of disorder in $\alpha$-chitin now becomes apparent. For the A setting, the intrachain $\mathrm{O}-\mathrm{H} \cdots \mathrm{O}$ bond is shorter than the interchain $\mathrm{N}-\mathrm{H} \cdots \mathrm{O}$ link; it is the other way around for $\mathbf{B}$. The mixed model, ABAB, contains two distinct $\mathrm{HBs}$ that locally resemble the respective pure model almost perfectly (to within $\sim 0.02 \AA$ ); this mixed model is visualized in Figure 3 . Apparently, the strong HBs in $\alpha$-chitin can be modeled with comparably small unit cells, even in the presence of disorder. 
This provides an important justification for using small and discrete approximants to a complex biomolecule.

The HB connectivity, and the role of the disordered groups, can best be seen when viewing the chains from above (down the $b$-axis); such drawings are provided in Figures $4 \mathrm{~b}, \mathrm{c}$. One is

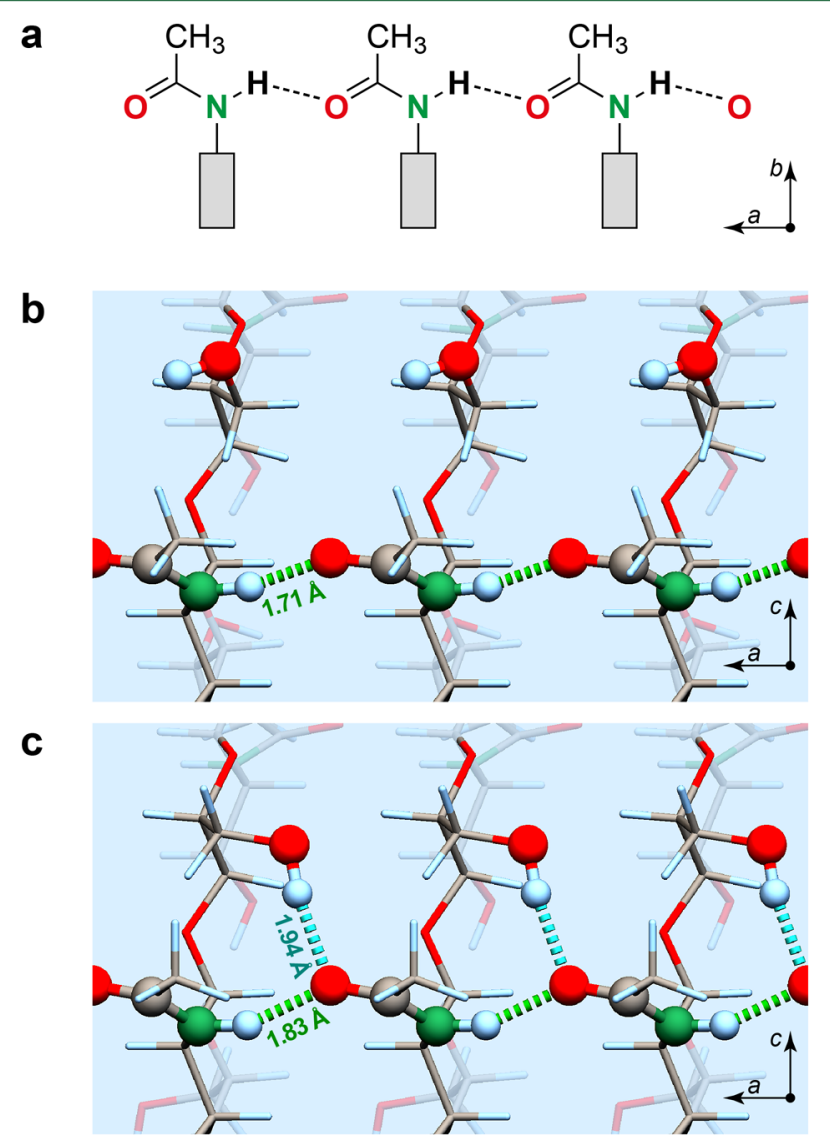

Figure 4. (a) Sketch of the $\mathrm{HB}$ chain along the $a$-axis that connects parallel strands in $\alpha$-chitin. The running direction of the chains is perpendicular to the paper plane. (b) Top view of a layer from the optimized BBBB model, emphasizing the cooperative $\mathrm{HB}$ chain formed by the acetylamine moieties. The hydroxymethyl groups do not take part in this chain. (c) Same for the AAAA model. Here, the hydroxymethyl groups form additional HBs with the acetyl oxygen; as a result, the $\mathrm{N}-\mathrm{H} \cdots \mathrm{O}$ distance increases.

immediately tempted to suggest a competition: is it better to have an isolated but shorter $\mathrm{N}-\mathrm{H} \cdots \mathrm{O}$ bond (Figure $4 \mathrm{~b}$ ) or a longer one but with additional binding from the hydroxymethyl group (Figure 4c); does $\mathrm{HB}$ anticooperativity occur if multiple hydrogens connect to one acceptor? We will now address these questions from the energetic viewpoint.

Interaction Energies: 3D, 2D, and 1D. Supercell models in which a low-dimensional fragment is enclosed by artificial vacuum have become widely used tools in DFT-based materials simulations. They can likewise be used to explore molecular crystals by computationally "cleaving" the lattice apart and running total-energy computations for isolated structural fragments. ${ }^{11,26}$ We illustrate this procedure for $\alpha$-chitin in Figure 5a. The characteristic building unit in this case is a onedimensional chain along the $c$-axis. The primary interchain linkage via the acetylamine groups extends along a (Figure 4) and such an alignment with the crystallographic axes makes it easy to identify lower-dimensional fragments without requiring
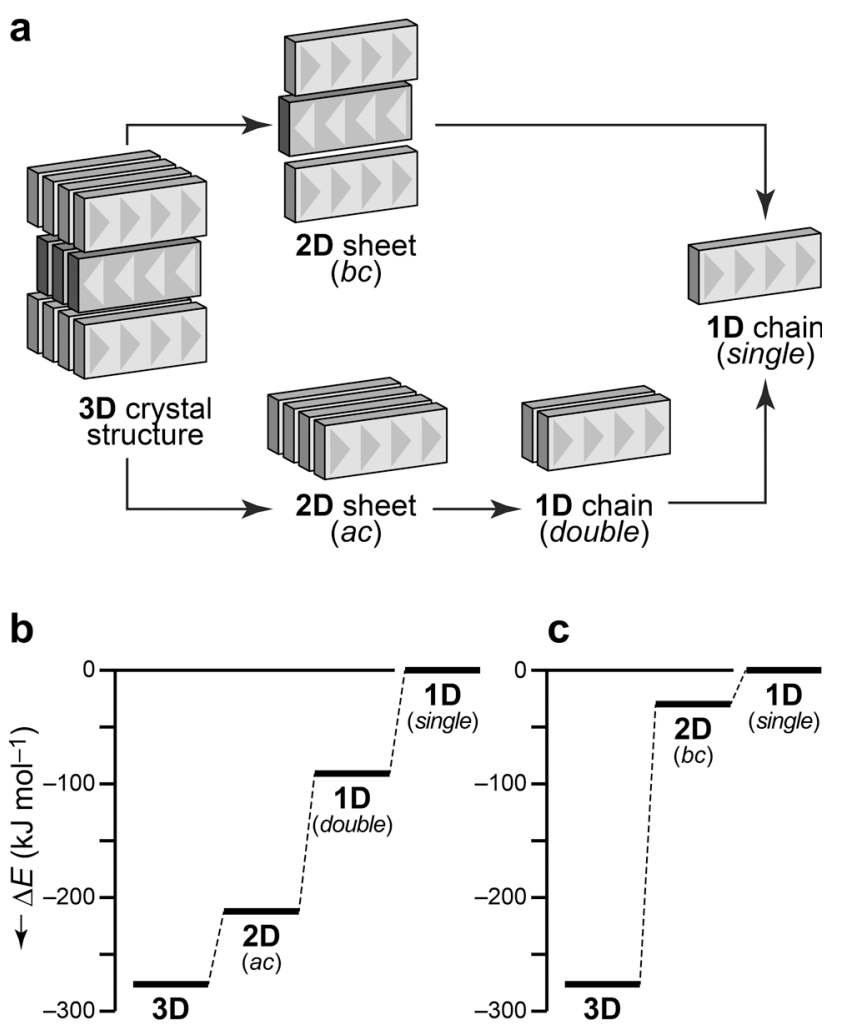

Figure 5. (a) Deconstructing the "building units" in crystalline $\alpha$-chitin (cf. Figure 1b) along different pathways, following a scheme outlined in ref $11 \mathrm{c}$. $(\mathrm{b}, \mathrm{c})$ Computed, dimensionally resolved energy profiles for the AAAA model. Interchain interaction energies $\Delta E$ have been evaluated according to eq 1 ; the lower, the more stable, and the single chain forms the energy zero.

unit-cell transformations. $^{26 a}$ In what follows, we compute interaction energies $\Delta E$ relative to an isolated chain

$$
\Delta E=\frac{1}{N}\left[E_{\mathrm{SC}}-N E_{1 \mathrm{D}, \mathrm{s}}\right]
$$

where $E_{\mathrm{SC}}$ and $E_{1 \mathrm{D}, \mathrm{s}}$ are DFT-D results for the respective supercell model and for a single $1 \mathrm{D}$ chain, respectively, and there are $N$ chitobiose units in the supercell. Depending on the hydroxymethyl configuration present in the model $(\mathbf{A} / \mathbf{B})$, the appropriate $1 \mathrm{D}$ counterpart is chosen as reference in all cases.

The resulting energy profile is shown in Figure $5 b$, exemplarily so for AAAA. The 3D crystal is (and must be) most stable with an energy gain of $\sim 275 \mathrm{~kJ} \mathrm{~mol}^{-1}$ relative to the constituent chains. The $2 \mathrm{D}(\mathrm{ac})$ sheet is held together by the aforementioned acetylamine links and is still stabilized by over $200 \mathrm{~kJ} \mathrm{~mol}^{-1}$; the energy then gets gradually higher, and the final step corresponds to a simple binding-energy computation between two isolated $1 \mathrm{D}$ chains.

It is interesting to compare this result with a previous $\mathrm{MD}$ study by Beckham and Crowley ${ }^{19}$ where the distribution of HBs in $\alpha$-chitin had been assessed in a principally complementary way, namely, as a statistical average; while Figure 5 displays energies, MD on large models reveals the likelihood of observing particular HBs. In the cited work, Beckham and Crowley quantified the directionally resolved occurrence of HBs for a particular $\alpha$-chitin chain inside the bulk. The authors found $19.1 \pm 0.9$ "self-" $\mathrm{HBs},{ }^{19}$ which correspond to the intrachain $\mathrm{O}-\mathrm{H} \cdots \mathrm{O}$ links (Figure 2). The latter, however, are an intrinsic feature of the $1 \mathrm{D}$ single chain, 
and thus they are not reflected in our energetic analysis (eq 1). Moreover, Beckham and Crowley arrived at $33.9 \pm 1.9 \mathrm{HBs}$ involving the acetylamine links (Figure 4) whereas only a very minor degree $(0.4 \pm 0.3)$ of hydrogen bonding was observed between the $2 \mathrm{D}$ sheets. In fact, Figure $5 \mathrm{~b}$ represents a more extreme case of this; there is no $\mathrm{HB}$ between the $2 \mathrm{D}$ sheets in our AAAA model, and the energy gain upon going from $3 \mathrm{D}$ to $2 \mathrm{D}$ in Figure $5 \mathrm{~b}$ thus reflects the dispersive forces between the polymer strands.

The next step, breaking up the $2 \mathrm{D}$ sheet, requires more than $100 \mathrm{~kJ} \mathrm{~mol}^{-1}$; this is in line with the high abundance of HBs in the MD model of ref 19. In hindsight, the latter work also supports both the mixing of $\mathbf{A}$ and $\mathbf{B}$ inferred from experiment and the preference for $\mathbf{A}$ found in DFT-D energies (Table 1); the simulated ratio of acetylamine versus intrachain $\mathrm{HBs}$ in ref 19 is $33.9 / 19.1=1.77$, whereas this ratio would be 1.0 in the pure BBBB model (Figure 4b), and 2.0 in AAAA (Figure 4c). Indeed, a combined theoretical view comprising both shorter and larger length scales does seem very rewarding to study biomacromolecules.

Let us return to the dimensionally resolved analyses of Figure 5. In principle, there is another option to cleave a $2 \mathrm{D}$ sheet from the crystal, namely, by "cutting" the acetylamine links. Chemically, however, doing so seems intuitively unfavorable, and the computed energy profile (Figure 5c) confirms this: going from the $3 \mathrm{D}$ crystal to an artificial $2 \mathrm{D}(b c)$ layer costs over $250 \mathrm{~kJ} \mathrm{~mol}^{-1}$ in stabilization, and the latter twodimensional fragment is only slightly more favorable than the corresponding isolated $1 \mathrm{D}$ chains; the situation here is clearly much different from the 2D (ac) motif. Cleavage of $\alpha$-chitin should thus proceed between the ac planes and leave the $\mathrm{N}-$ $\mathrm{H} \cdots \mathrm{O}$ bridges intact. Note, however, that the stepwise enzymatic decrystallization of a single chain may proceed along different routes, and that the several facets needed to describe this process cannot be addressed in the framework used here.

For a more comprehensive picture, we have collected dimensionally resolved interaction energies $\Delta E$ (eq 1) but now start from all three representative crystal models under consideration. We have thus added $\mathbf{A B A B}$ and $\mathbf{B B B B}$ to the investigation, and the results are compiled in Figure 6.

The differences between the models are most pronounced for the $2 \mathrm{D}(a c)$ sheets, and the all-A configuration is most strongly bound. This appears to be a direct consequence of the two possible $\mathrm{HB}$ arrangements in the interchain acetylaminemediated links (Figure $5 \mathrm{~b}$ ); if the hydroxymethyl group takes part in this interchain $\mathrm{HB}$ (i.e., is in configuration A), the overall energetic situation is most favorable. The elongation of the adjacent $\mathrm{H} \cdots \mathrm{O}$ contact (Figure 4c) does not change this. Figure 6 also allows us to probe the energetic cooperativity (or nonadditivity) of the $\mathrm{HBs}$ along the $a$ direction; comparing the $1 \mathrm{D}$ (double) to the 2D (ac) fragments, the amount of interchain HBs per chitobiose unit doubles, but $\Delta E$ is more than doubled, which shows mutual stabilization. If the ratio were below 2, it would evidence energetic anticooperativity but this is not the case for any of the three structural models. More details on this particular approach may be found in ref $11 \mathrm{c}$.

An interesting detail is observed when computing $\Delta E$ for the 2D (bc) fragments (right-hand side of Figure 6). While these fragments are overall clearly less likely to form than $2 \mathrm{D}(a c)$, they do among themselves show a behavior that is markedly different from the other fragments, and the all-A model this time comes out least favorable among the three $2 \mathrm{D}(b c)$ sheets

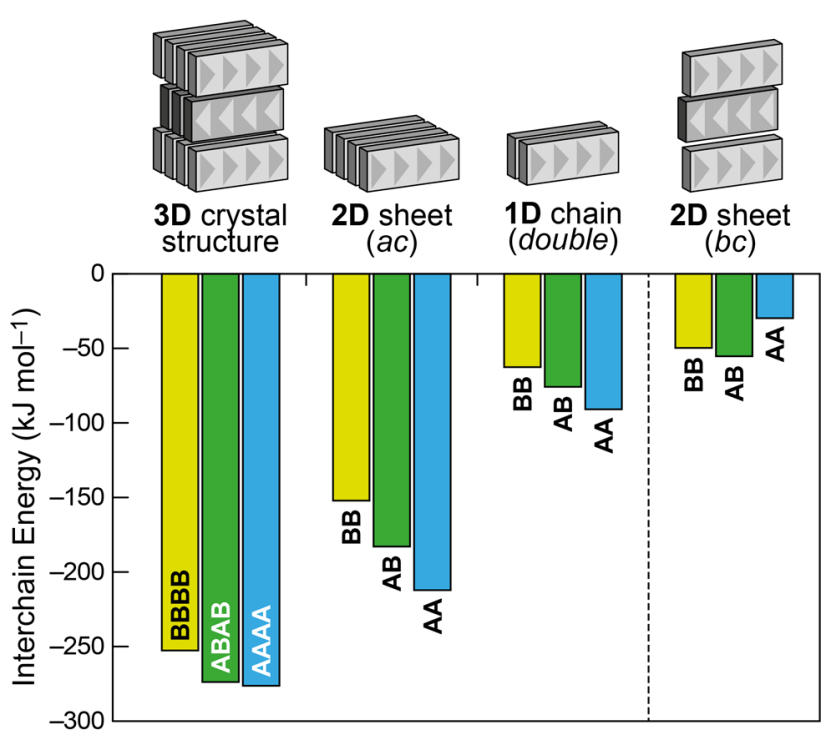

Figure 6. Interchain energies as defined in the text (eq 1), all relative to isolated $1 \mathrm{D}$ chains that are set as the energy zero. These energies have been computed for three different $\alpha$-chitin crystal models and for lower-dimensional fragments derived from them. (Note that the energy differences between the 3D structures are not identical to those listed in Table 1; this is so by definition, as different $1 \mathrm{D}$ fragments are used for normalization).

we have studied. The BB $(b c)$ sheet is marginally less stable than $\mathbf{A B}(b c)$ in computed overall interaction energies, but it is by far not as strongly disfavored as the other all-B structural motifs are.

Taking a comprehensive look at Figure 6, one may conclude that the ratio of intrasheet (defined as being within the strongly bound ac plane) and intersheet energies is what sets the three discrete models apart. With DFT-D energies at hand, one can now directly quantify this ratio (Figure 7a): the overall contribution from intersheet interactions almost doubles depending on whether all-A or all-B planes are stacked on top of each other.
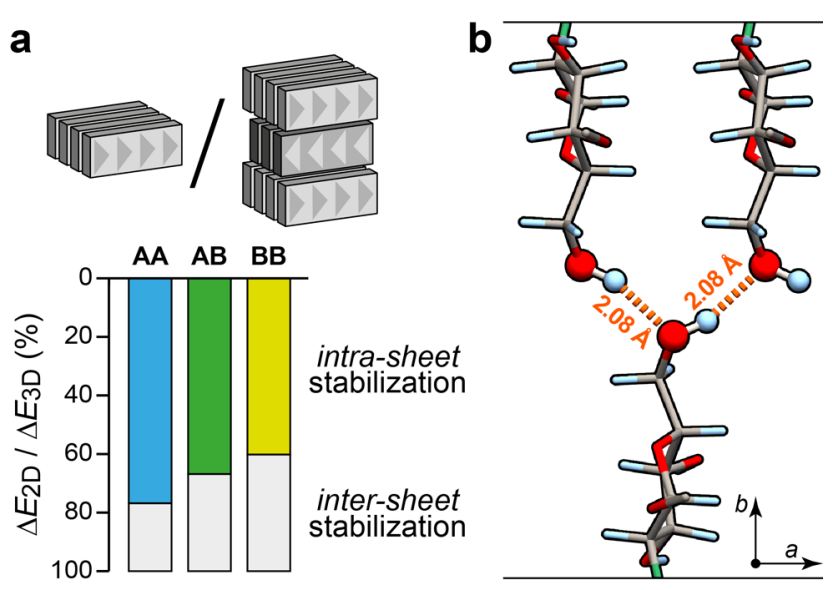

Figure 7. (a) Ratios of interaction energies for 2D (ac) versus 3D structures (see sketch); the percentage of intrasheet stabilization so estimated is given by colored bars. The remaining fraction is attributed to intersheet contributions (gray bars). (b) Fragment from the optimized BBBB crystal structure in which the intersheet stabilization is strongest; this is due to additional HBs formed by hydroxymethyl groups in "B" configuration, as visualized here. 
Why is that so? A closer look at the fully optimized BBBB model structure provides an answer and the "missing link" (Figure $7 \mathrm{~b}$ ): there are additional HBs between the ac planes, which can only form if the hydroxymethyl group is " $\mathbf{B}$ "-oriented and points upward, away from the chain (Figures 2-3). A comparable situation is encountered in the ABAB model but here only every second hydroxymethyl group (namely, the Boriented ones) forms an $\mathrm{HB}$ to a neighboring (A-oriented) one. In this case, the $\mathrm{H} \cdots \mathrm{O}$ distance is even shorter $(1.86 \AA)$, albeit the intersheet $\mathrm{HBs}$ are isolated in $\mathbf{A B A B}$, and forms an extended motif in BBBB (Figure 7b). Neither of these options is possible in AAAA.

Such reinforcement of bonding in $b$ direction upon leaving the ground state has also been seen in the conformational study of ref 18. Indeed, one may assume that it is this formation of additional $\mathrm{HBs}$ (Figure $7 \mathrm{~b}$ ) that causes an appreciable amount of B-oriented hydroxymethyl groups to be present in the crystal. Thus, crystallographic disorder ultimately enables the three-dimensional linking that is at the heart of the material's stability. Necessarily, this argument is a qualitative one because DFT-D simulations at this length scale provide small, discrete approximants to the macroscopic system; this is somewhat reminiscent of a recent study on salient bonding features of cellulose, in which the authors restricted themselves to a cellobiose molecule as the essential, isolated "building block" ${ }^{40}$ There, and here, it is apparent that small chemical models are able to capture several important features of biomolecular systems no matter what.

\section{CONCLUSIONS}

We have explored intermolecular interactions in $\alpha$-chitin, focusing on the nature and strength of hydrogen bonding, which is a central theme in the study of biomacromolecules. Our atomistic DFT-D study confirms previous propositions of interchain links via the acetylamine moieties, caused by strongly cooperative $\mathrm{N}-\mathrm{H} \cdots \mathrm{O}=\mathrm{C}$ bonds. A neighboring hydroxymethyl group can connect to the same acceptor, which is energetically favorable albeit it increases the $\mathrm{H} \cdots \mathrm{O}$ distances; no evidence for energetic anticooperativity is found. The overall interchain stabilization in $\alpha$-chitin exceeds $250 \mathrm{~kJ} \mathrm{~mol}^{-1}$, regardless of the particular model used. The alternative discrete arrangements for the orientationally disordered hydroxymethyl moieties have allowed us to address the role of disorder for intra- and interlayer stabilization: ultimately, the material needs both $\mathrm{HB}$ motifs to stabilize its rigid $3 \mathrm{D}$ structure, and this can only be achieved by disorder. In this sense, $\alpha$-chitin differs from cellulose where weaker and nondirectional vdW bonding holds the polymer strands together. On the basis of the present work, it will now be possible to further explore macroscopic properties of $\alpha$-chitin, and the extension to a wide range of chemically related biomaterials does seem rewarding.

\section{ASSOCIATED CONTENT}

\section{S Supporting Information}

The Supporting Information is available free of charge on the ACS Publications website at DOI: 10.1021/acs.biomac.5b01653.

Results of DFT-D computations on the allomorphic form $\beta$-chitin and Cartesian coordinates of supercell models employed. (PDF)

\section{AUTHOR INFORMATION}

\section{Corresponding Authors}

*E-mail: ullrich.englert@ac.rwth-aachen.de. Fax: +49 24180 92288 (U.E.).

*E-mail: drons@HAL9000.ac.rwth-aachen.de. Fax: +49 24180 92642 (R.D.).

\section{Author Contributions}

The manuscript was written through contributions of all authors. All authors have given approval to the final version of the manuscript.

Notes

The authors declare no competing financial interest.

\section{ACKNOWLEDGMENTS}

The authors gratefully acknowledge computational resources provided by JARA-HPC at RWTH Aachen University (project jara0069).

\section{REFERENCES}

(1) (a) Rinaudo, M. Prog. Polym. Sci. 2006, 31, 603-632. (b) Pillai, C. K. S.; Paul, W.; Sharma, C. P. Prog. Polym. Sci. 2009, 34, 641-678. (c) Wysokowski, M.; Petrenko, I.; Stelling, A. L.; Stawski, D.; Jesionowski, T.; Ehrlich, H. Polymers 2015, 7, 235-265.

(2) Minke, R.; Blackwell, J. J. Mol. Biol. 1978, 120, 167-181.

(3) Sikorski, P.; Hori, R; Wada, M. Biomacromolecules 2009, 10, $1100-1105$

(4) Kameda, T.; Miyazawa, M.; Ono, H.; Yoshida, M. Macromol. Biosci. 2005, 5, 103-106.

(5) Kumirska, J.; Czerwicka, M.; Kaczyński, Z.; Bychowska, A.; Brzozowski, K.; Thöming, J.; Stepnowski, P. Mar. Drugs 2010, 8, $1567-1636$

(6) (a) Keten, S.; Buehler, M. J. Nano Lett. 2008, 8, 743-748. (b) Keten, S.; Xu, Z.; Ihle, B.; Buehler, M. J. Nat. Mater. 2010, 9, 359367. (c) Nikolov, S.; Petrov, M.; Lymperakis, L.; Friák, M.; Sachs, C.; Fabritius, H.-O.; Raabe, D.; Neugebauer, J. Adv. Mater. 2010, 22, 519526.

(7) (a) Azizi Samir, M. A. S.; Alloin, F.; Dufresne, A. Biomacromolecules 2005, 6, 612-626. (b) Kim, J.; Yun, S.; Ounaies, Z. Macromolecules 2006, 39, 4202-4206. (c) Moon, R. J.; Martini, A.; Nairn, J.; Simonsen, J.; Youngblood, J. Chem. Soc. Rev. 2011, 40, 3941-3994. (d) Lin, N.; Huang, J.; Dufresne, A. Nanoscale 2012, 4, 3274-3294. (e) Sinko, R.; Qin, X.; Keten, S. MRS Bull. 2015, 40, $340-348$.

(8) Hobza, P. Acc. Chem. Res. 2012, 45, 663-672.

(9) (a) Dunitz, J. D.; Gavezzotti, A. Cryst. Growth Des. 2012, 12, 5873-5877. (b) Maloney, A. G. P.; Wood, P. A.; Parsons, S. CrystEngComm 2014, 16, 3867-3882.

(10) (a) Schmidtmann, M.; Farrugia, L. J.; Middlemiss, D. S.; Gutmann, M. J.; McIntyre, G. J.; Wilson, C. C. J. Phys. Chem. A 2009, 113, 13985-13997. (b) Contreras-García, J.; Johnson, E. R.; Keinan, S.; Chaudret, R.; Piquemal, J.-P.; Beratan, D. N.; Yang, W. J. Chem. Theory Comput. 2011, 7, 625-632. (c) Shishkina, A. V.; Zhurov, V. V.; Stash, A. I.; Vener, M. V.; Pinkerton, A. A.; Tsirelson, V. G. Cryst. Growth Des. 2013, 13, 816-828.

(11) (a) Morrison, C. A.; Siddick, M. M. Chem.-Eur. J. 2003, 9, 628-634. (b) Morrison, C. A.; Siddick, M. M. Angew. Chem., Int. Ed. 2004, 43, 4780-4782. (c) Hoepfner, V.; Deringer, V. L.; Dronskowski, R. J. Phys. Chem. A 2012, 116, 4551-4559.

(12) Brandenburg, J. G.; Hochheim, M.; Bredow, T.; Grimme, S. J. Phys. Chem. Lett. 2014, 5, 4275-4282.

(13) (a) Bučko, T.; Tunega, D.; Ángyán, J. G.; Hafner, J. J. Phys. Chem. A 2011, 115, 10097-10105. (b) Li, Y.; Lin, M.; Davenport, J. M. J. Phys. Chem. C 2011, 115, 11533-11539. (c) Lee, C. M.; Mohamed, N. M. A.; Watts, H. D.; Kubicki, J. D.; Kim, S. H. J. Phys. Chem. B 2013, 117, 6681-6692. 
(14) Nishiyama, Y.; Johnson, G. P.; French, A. D.; Forsyth, V. T.; Langan, P. Biomacromolecules 2008, 9, 3133-3140.

(15) Qian, X.; Ding, S.-Y.; Nimlos, M. R.; Johnson, D. K.; Himmel, M. E. Macromolecules 2005, 38, 10580-10589.

(16) (a) Bellesia, G.; Asztalos, A.; Shen, T.; Langan, P.; Redondo, A.; Gnanakaran, S. Acta Crystallogr., Sect. D: Biol. Crystallogr. 2010, 66, 1184-1188. (b) Beckham, G. T.; Matthews, J. F.; Peters, B.; Bomble, Y. L.; Himmel, M. E.; Crowley, M. F. J. Phys. Chem. B 2011, 115, 4118-4127. (c) Payne, C. M.; Himmel, M. E.; Crowley, M. F.; Beckham, G. T. J. Phys. Chem. Lett. 2011, 2, 1546-1550.

(17) Uto, T.; Mawatari, S.; Yui, T. J. Phys. Chem. B 2014, 118, 93139321.

(18) Petrov, M.; Lymperakis, L.; Friák, M.; Neugebauer, J. Biopolymers 2013, 99, 22-34.

(19) Beckham, G. T.; Crowley, M. F. J. Phys. Chem. B 2011, 115, $4516-4522$.

(20) Jin, K.; Feng, X.; Xu, Z. BioNanoScience 2013, 3, 312-320.

(21) Keen, D. A.; Goodwin, A. L. Nature 2015, 521, 303-309.

(22) Siegrist, T.; Jost, P.; Volker, H.; Woda, M.; Merkelbach, P.; Schlockermann, C.; Wuttig, M. Nat. Mater. 2011, 10, 202-208.

(23) (a) Snyder, G. J.; Christensen, M.; Nishibori, E.; Caillat, T.; Iversen, B. B. Nat. Mater. 2004, 3, 458-463. (b) Wang, H.; LaLonde, A. D.; Pei, Y.; Snyder, G. J. Adv. Funct. Mater. 2013, 23, 1586-1596. (c) Siegert, K. S.; Lange, F. R. L.; Sittner, E. R.; Volker, H.; Schlockermann, C.; Siegrist, T.; Wuttig, M. Rep. Prog. Phys. 2015, 78, 013001.

(24) Frost, J. M.; Butler, K. T.; Brivio, F.; Hendon, C. H.; van Schilfgaarde, M.; Walsh, A. Nano Lett. 2014, 14, 2584-2590.

(25) Hirsch, T. K.; Ojamäe, L. J. Phys. Chem. B 2004, 108, 1585615864.

(26) (a) Deringer, V. L.; Pan, F.; George, J.; Müller, P.; Dronskowski, R.; Englert, U. CrystEngComm 2014, 16, 135-138. (b) George, J.; Deringer, V. L.; Dronskowski, R. J. Phys. Chem. A 2014, 118, 31933200. (c) George, J.; Deringer, V. L.; Dronskowski, R. Inorg. Chem. 2015, 54, 956-962.

(27) Steiner, T. Angew. Chem., Int. Ed. 2002, 41, 48-76.

(28) (a) Qian, X. Mol. Simul. 2008, 34, 183-191. (b) Parthasarathi, R.; Bellesia, G.; Chundawat, S. P. S.; Dale, B. E.; Langan, P.; Gnanakaran, S. J. Phys. Chem. A 2011, 115, 14191-14202.

(29) Perdew, J. P.; Burke, K.; Ernzerhof, M. Phys. Rev. Lett. 1996, 77, 3865-3868.

(30) Grimme, S.; Antony, J.; Ehrlich, S.; Krieg, H. J. Chem. Phys. 2010, 132, 154104.

(31) (a) Johnson, E. R.; Becke, A. D. J. Chem. Phys. 2006, 124, 174104. (b) Grimme, S.; Ehrlich, S.; Goerigk, L. J. Comput. Chem. 2011, 32, 1456-1465.

(32) (a) Goerigk, L.; Grimme, S. Phys. Chem. Chem. Phys. 2011, 13, 6670-6688. (b) Goerigk, L.; Kruse, H.; Grimme, S. ChemPhysChem 2011, 12, 3421-3433. (c) Moellmann, J.; Grimme, S. J. Phys. Chem. C 2014, 118, 7615-7621.

(33) (a) Nishiyama, Y.; Noishiki, Y.; Wada, M. Macromolecules 2011, 44, 950-957. (b) Sawada, D.; Nishiyama, Y.; Langan, P.; Forsyth, V. T.; Kimura, S.; Wada, M. Biomacromolecules 2012, 13, 288-291.

(34) (a) Kresse, G.; Hafner, J. Phys. Rev. B: Condens. Matter Mater. Phys. 1993, 47, 558-561. (b) Kresse, G.; Furthmüller, J. Phys. Rev. B: Condens. Matter Mater. Phys. 1996, 54, 11169-11186. (c) Kresse, G.; Furthmüller, J. Comput. Mater. Sci. 1996, 6, 15-50. (d) Kresse, G.; Joubert, D. Phys. Rev. B: Condens. Matter Mater. Phys. 1999, 59, 17581775.

(35) Blöchl, P. E. Phys. Rev. B: Condens. Matter Mater. Phys. 1994, 50, 17953-17979.

(36) Monkhorst, H. J.; Pack, J. D. Phys. Rev. B 1976, 13, 5188-5192.

(37) Birch, F. Phys. Rev. 1947, 71, 809-824.

(38) Dunitz, J. D.; Gavezzotti, A. Angew. Chem., Int. Ed. 2005, 44, 1766-1787.

(39) (a) Deringer, V. L.; Hoepfner, V.; Dronskowski, R. Cryst. Growth Des. 2012, 12, 1014-1021. (b) Madsen, A. Ø.; Hoser, A. A. J. Appl. Crystallogr. 2014, 47, 2100-2104.
(40) Loerbroks, C.; Rinaldi, R.; Thiel, W. Chem.-Eur. J. 2013, 19, $16282-16294$. 